Supporting Information

\title{
Integration of Multiplexed Microfluidic Electrokinetic Concentrators with a Morpholino Microarray via Reversible Surface Bonding for Enhanced DNA Hybridization
}

Diogo Martins ${ }^{\S}, \mathrm{Xi} \mathrm{We}^{\S, \dagger}$, Rastislav Levicky ${ }^{\dagger}$, and Yong-Ak Song ${ }^{\S, \dagger, ~ * ~}$

§Division of Engineering, New York University Abu Dhabi, P.O. Box 129188, Abu Dhabi, United Arab Emirates, ${ }^{\dagger}$ Department of Chemical and Biomolecular Engineering, New York University Tandon School of Engineering, Brooklyn, New York 11201, United States

*Corresponding author: E-mail: rafael.song@nyu.edu. Phone: +971-2-628-478. Fax: +971-2-659-0794.

\begin{abstract}
Supporting information provides: a video showing the automatic PEDOT:PSS printing process on PDMS channel, 3D confocal videos of the DNA plug during concentration experiments, confocal data of the vertical concentration profile of the plug in microfluidic concentrators with PEDOT:PSS at the top and bottom of the microchannel, estimation of the $\mathrm{pH}$ shift during ET concentration a 5-channel simultaneous DNA concentration video, and a measurement of the channel-tochannel and device-to-device variation.
\end{abstract}




\section{S1. PEDOT:PSS printing on the PDMS}

Video S-1 shows the PEDOT:PSS printing step in 5 parallel PDMS microchannels. Prior to the printing step, the PDMS microchannels were rendered hydrophilic via confined corona plasma through the microchannels. The microchannels were then mounted onto the microplotter and the center position of each circular pattern in the microchannel is saved for subsequent access. The glass micropipette was then filled with PEDOT:PSS solution and sequentially driven to each one of the saved positions for in-channel membrane deposition (Video S-1). During this last step, the localized in-channel hydrophilicity allowed the PEDOT:PSS solution to spread inside the circular pattern and did not overflow into the connecting microchannel or over the channel edge which could compromise the reversible bonding between the PDMS chip and the substrate. 


\section{S2. 3D confocal videos of the DNA plug}

The microchannel volume was vertically scanned in $2 \mu \mathrm{m}$ step size. Video S-2 [last frame showed in Fig S-1 (a)], shows the formation of a DNA plug on a straight microchannel just next to a PEDOT:PSS membrane printed on top of the channel with no MOs. Video S-3 shows a DNA plug next to a PEDOT:PSS membrane printed at the bottom of a microchannel without MOs. Video S-4 shows a DNA plug next to a PEDOT:PSS membrane printed at the top of a microchannel with $\mathrm{MO}$ spots printed. The initial DNA concentration for all three experiments was $100 \mathrm{nM}$ and the applied voltage was $75 \mathrm{~V}$. These videos are all compressed time videos (15 min. total, real time). In these videos, the DNA plug starts forming near the membrane and the maximum concentration region of the plug slowly moves away from the membrane with time. In the videos, it is clear that the vertical concentration profile is not uniform and is highest at the bottom of the microchannel (colors representing different fluorescence intensities, from the highest to the lowest intensity: white, yellow, purple, blue).

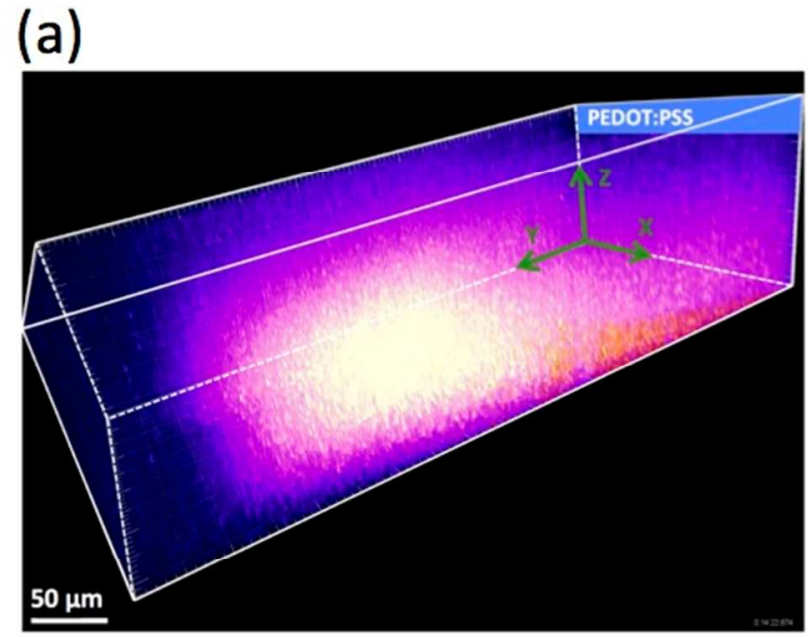

(b)

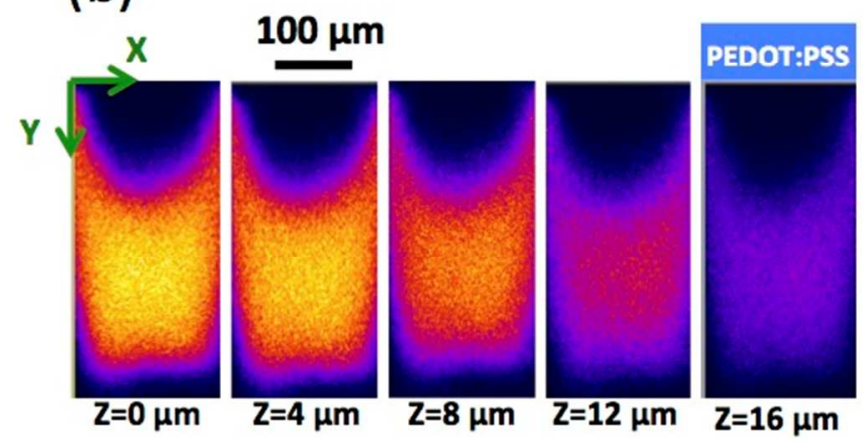


Figure S-1: (a) 3D reconstruction from the confocal fluorescence microscopy images in (b) of the DNA plug in a PDMS microchannel with a PEDOT:PSS membrane printed on top of the channel. The membrane boundary matches with the rightmost vertical facet of the rectangular prism domain in (a). Note: in (a) for clarity, the z-axis is magnified by $10 \times$ relative to the $y$ - and $x$-axis. 
S3. Vertical concentration profile for $100 \mathrm{nM}$ DNA concentration on a deactivated superaldehyde glass slide without MO

Figure S-2 depicts the fluorescence intensity distribution along the vertical axis of the microchannel. The initial sample concentration was $100 \mathrm{nM}$. The imaging conditions used for $100 \mathrm{nM}$ were $5 \%$ for laser power and $\mathrm{HV}=702 \mathrm{~V}$ for photomultiplier voltage.

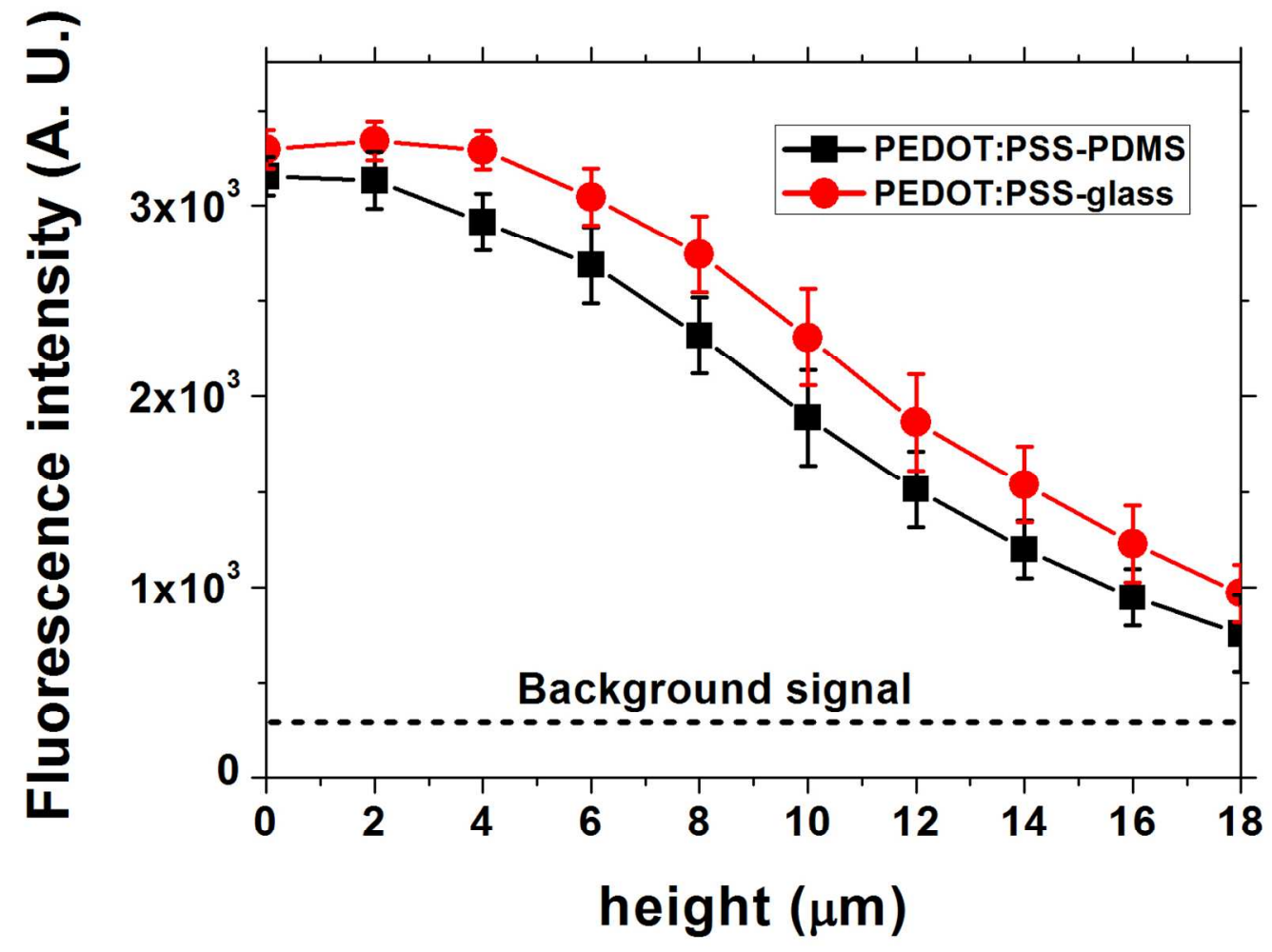

Figure S-2: Vertical intensity profile of the DNA plug as a function of the microchannel height, after applying $75 \mathrm{~V}$ across the microchannels for 15 min. These measurements were performed for devices with the PEDOT:PSS membrane at the top (squares, PEDOT:PSS-PDMS) of the microchannel and at the bottom (dots, PEDOT:PSS-glass). The results show that the membrane position does not affect significantly the plug profile when the initial DNA sample concentration was $100 \mathrm{nM}$. However, the fluorescence signal intensity was higher for PEDOT:PSS-glass devices than for PEDOT:PSS-PDMS devices. 
S4. Vertical concentration profile for $100 \mathrm{nM}$ DNA concentration on a deactivated superaldehyde glass slide with MO probes

Figure S-3 depicts the fluorescence intensity distribution along the vertical axis of microchannels with $\mathrm{MO}$ spots printed on the superaldehyde glass substrate. The initial DNA concentration was $100 \mathrm{nM}$. The imaging conditions used were $5 \%$ for laser power and $\mathrm{HV}=702 \mathrm{~V}$ for photomultiplier voltage.

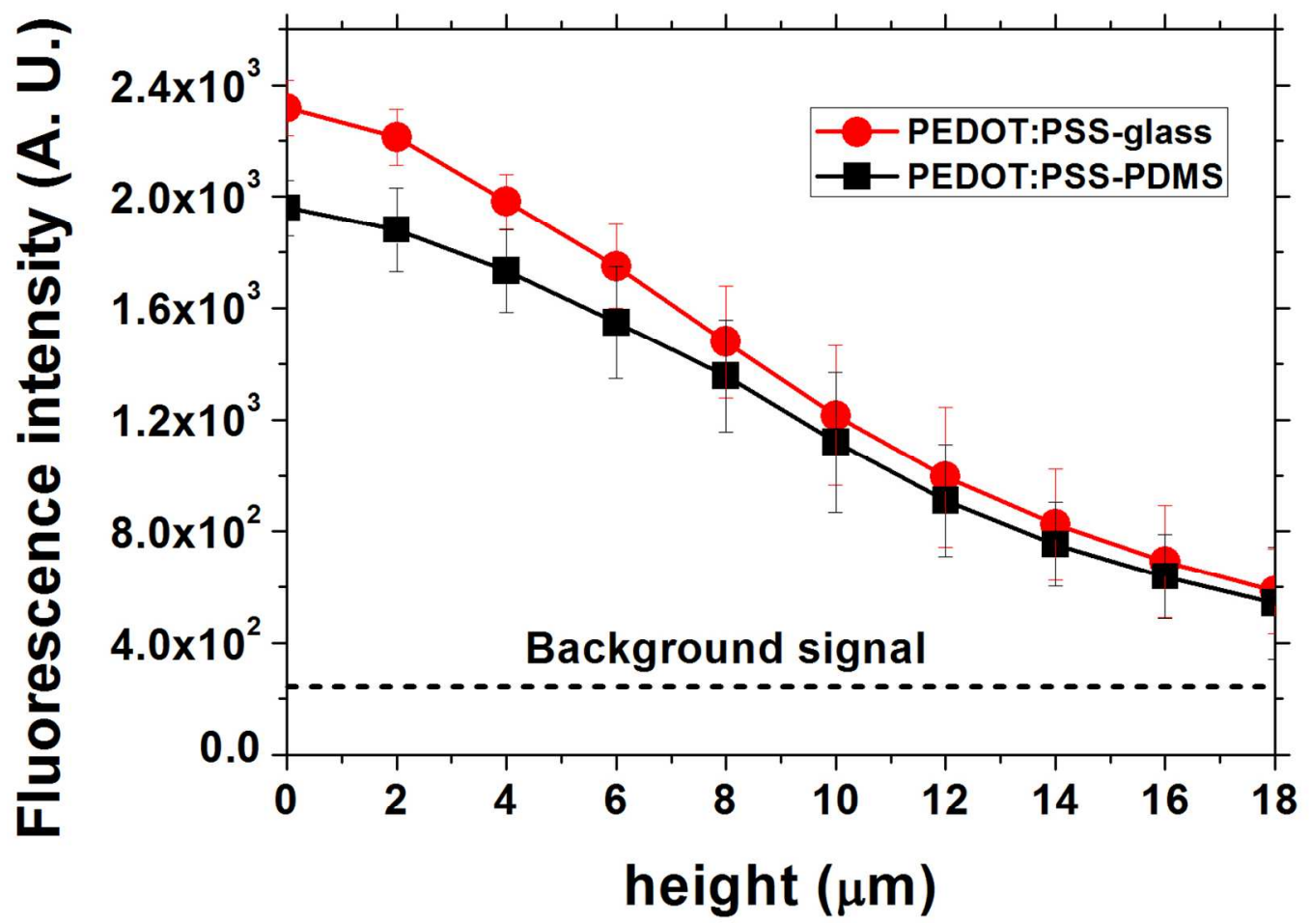

Figure S-3: Vertical intensity profile of the DNA plug as a function of the microchannel height, after applying $75 \mathrm{~V}$ across the microchannels for 15 min, during an MO-DNA hybridization experiment with an initial DNA concentration of $100 \mathrm{nM}$. These measurements were performed for devices with the PEDOT:PSS membrane at the top (squares, PEDOT:PSS-PDMS) of the microchannel and at the bottom (dots, PEDOT:PSS-glass). These results show that the MO spots did not affect significantly the plug signal intensity profiles. However, the data suggests that the presence of morpholino spots at the bottom of the microchannel slightly decreased the plug signal intensity in comparison with those devices with only deactivated superaldehyde glasses in agreement with the discussion on the electrophoretic mobilities of different substrates (compare with Fig. S2). 
S5. Vertical concentration profile for $10 \mathrm{nM}$ DNA concentration on a deactivated superaldehyde glass slide without MO

The imaging conditions used for $10 \mathrm{nM}$ were $25 \%$ for laser power and HV=790 V for photomultiplier voltage.

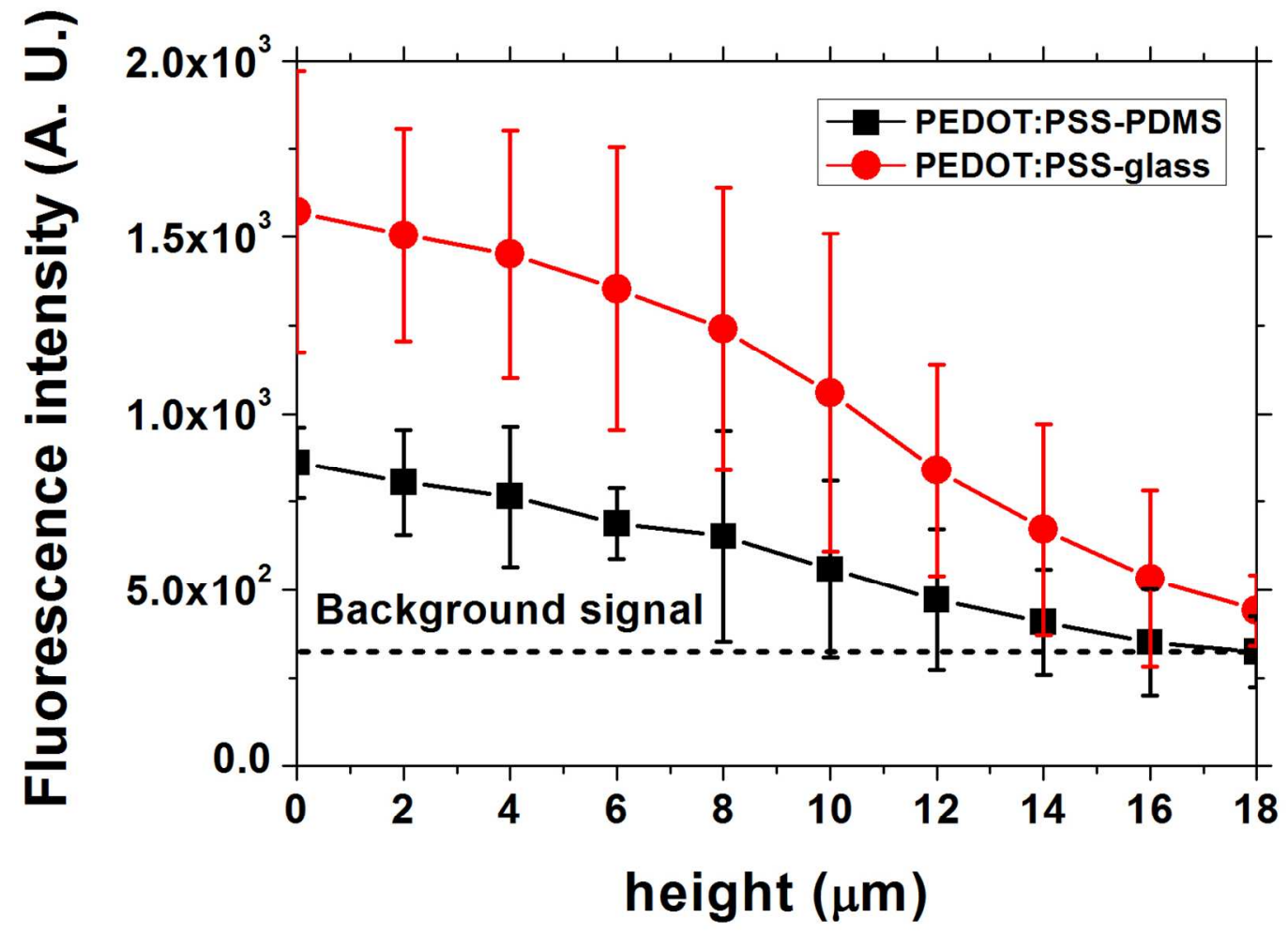

Figure S-4: Vertical intensity profile of the DNA plug as a function of the microchannel height, after applying $75 \mathrm{~V}$ across the microchannels for 15 min, for an initial DNA concentration of $10 \mathrm{nM}$. These measurements were performed for devices with the PEDOT:PSS membrane at the top (dots, PEDOT:PSS-glass) of the microchannel and at the bottom (squares, PEDOT:PSS-PDMS). The results show that, for an initial DNA sample of 10 $\mathrm{nM}$, the membrane position in the channel impacts significantly the DNA plug signal intensity. In fact, PEDOT:PSS-glass devices show the double of the background deduced fluorescence intensity at the surface when compared with the PEDOT:PSS-PDMS devices. 


\section{S6. $\mathrm{pH}$ shift in the plug region during preconcentration}

To estimate the $\mathrm{pH}$ variation during our electrokinetic concentration method, we separately concentrated two fluorescent dyes, Alexa Fluor 488 NHS Ester (ThermoFisher Scientific Cat. \# A-20000, MW 643.4, pH insensitive from $\mathrm{pH} 4$ to $\mathrm{pH} 10$ ) and fluorescein (ThermoFisher Scientific, Cat. \# F1300, MW 332.31, $\mathrm{pH}$ sensitive from $\mathrm{pH} 5$ to $\mathrm{pH} 9^{1,2}$ ), and then compared their fluorescence signal intensities as a measure of concentration factor [see Figure S-5 (a) below]. With Alexa Fluor 488, the fluorescence intensity of the plug increased linearly with time, whereas the fluorescence intensity of fluorescein increased linearly (the lower fluorescence intensities are due to the lower quantum yield of fluorescein in comparison to Alexa Fluor 488) for $8 \mathrm{~min}$. and reached a plateau after $12 \mathrm{~min}$. . The linear regression line is represented as a dash-dot green line. We attributed this deviation from the initial linearity to a $\mathrm{pH}$ drop in the concentration region and not to a decline in the rate of molecular accumulation, as it was only observed with $\mathrm{pH}$ sensitive fluorescein. In fact, for Alexa Fluor 488, the accumulation of molecules occurs at a constant rate due to the constant applied EOF, and because its fluorescence is not affected by a pH change. Therefore, the fluorescence intensity of the plug increased linearly with time, as observed. Since EOF dominates the transport of the charged species in the microchannel, fluorescein should accumulate at the same rate as Alexa Fluor 488 during concentration; consequently the observed fluorescence intensity plateau can be explained as a result of a $\mathrm{pH}$ drop during electrokinetic concentration. As mentioned earlier, fluorescein is $\mathrm{pH}$-sensitive and its fluorescence increases with higher $\mathrm{pH}$, as shown in Figure S-5 (b) below.

To calculate the $\mathrm{pH}$ drop during preconcentration, we first estimated the final fluorescein concentration (after $15 \mathrm{~min}$.) by using the electrokinetic concentration curve of Alexa Fluor 488 (Figure S-5 (a), blue squares) together with an Alexa Fluor 488 fluorescence intensity vs. concentration calibration curve [in Figure S-5 (a), Alexa Fluor 488 reference concentration lines are displayed as dashed lines]. As shown in Figure S-5 (a), we obtained a concentration factor of $\sim 600$ times (concentration increased from $0.1 \mu \mathrm{M}$ to $60 \mu \mathrm{M}$ ) for Alexa 488 dye after $15 \mathrm{~min}$. of electrokinetic concentration. By assuming the same concentration factor of 600 times for fluorescein, we calculated the final concentration of fluorescein to be $60 \mu \mathrm{M}$ starting from an initial concentration of $0.1 \mu \mathrm{M}$. By measuring the fluorescence signal intensity of $60 \mu \mathrm{M}$ fluorescein in $0.1 \mathrm{x}$ PBS titrated from pH 7.0 to pH 5.5 in 0.5 pH units step [see Figure S-5 (b)] and comparing the fluorescence intensity of the electrokinetically concentrated fluorescein dye after $15 \mathrm{~min}$. from Figure S-5 (a) to that of 60 
$\mu \mathrm{M}$ fluorescein between $\mathrm{pH} 5.5$ and 7.0 in Figure $\mathrm{S}-5$ (b), we determined a $\mathrm{pH}$ drop of $\sim 0.5 \mathrm{pH}$ units from the initial value of $\mathrm{pH} 7.0$ through a linear interpolation. The fact that the linear regression line of the fluorescence signal intensity for fluorescein without $\mathrm{pH}$ change [green line in Figure S-5 (a)] results in similar fluorescence signal intensity after $15 \mathrm{~min}$. of electrokinetic concentration compared to the fluorescence signal intensity of $60 \mu \mathrm{M}$ fluorescein at $\mathrm{pH} 7$ in Figure S-5 (b) validates our accurate estimation of 600 -fold increase of concentration for fluorescein. 
(a)

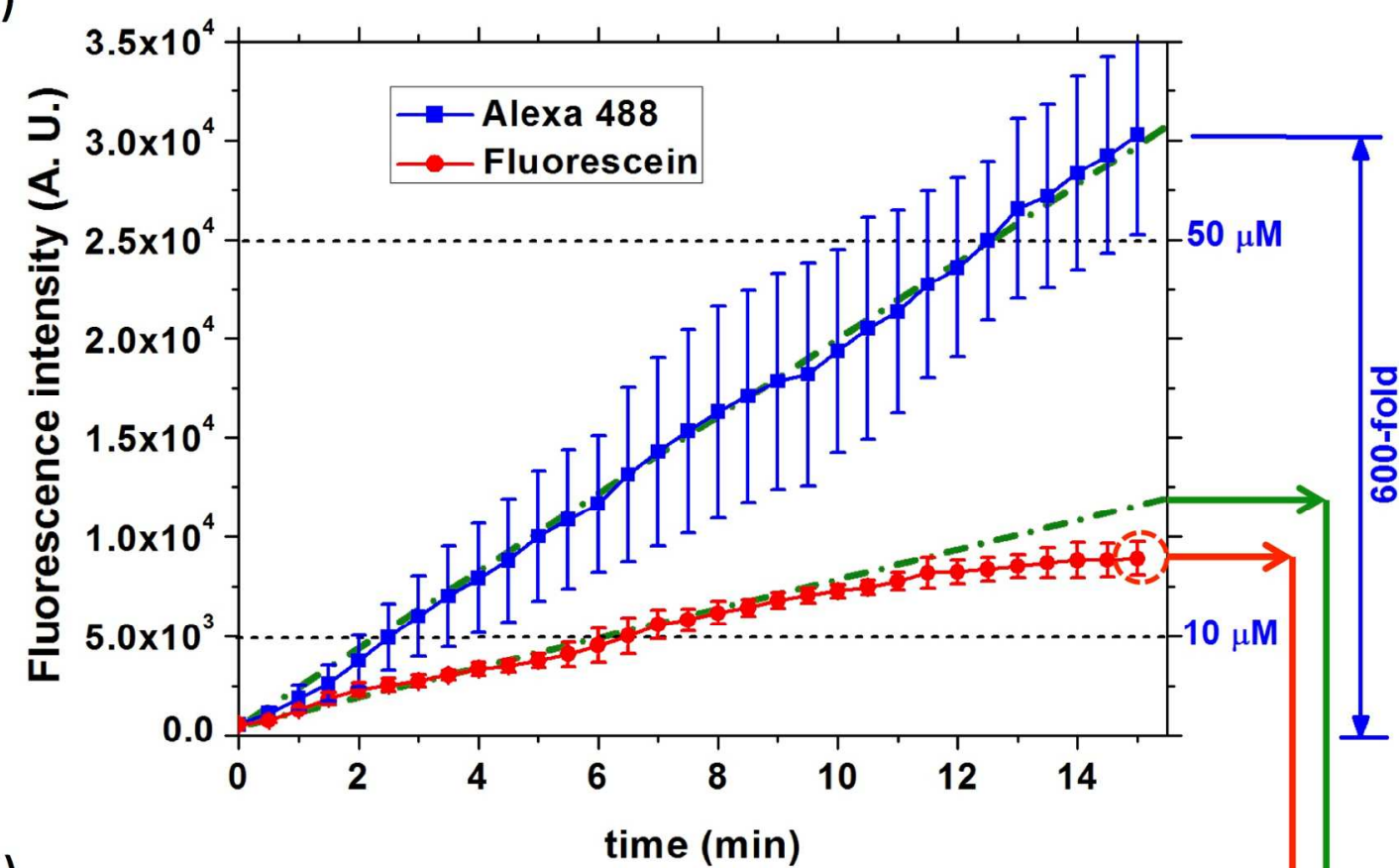

(b)

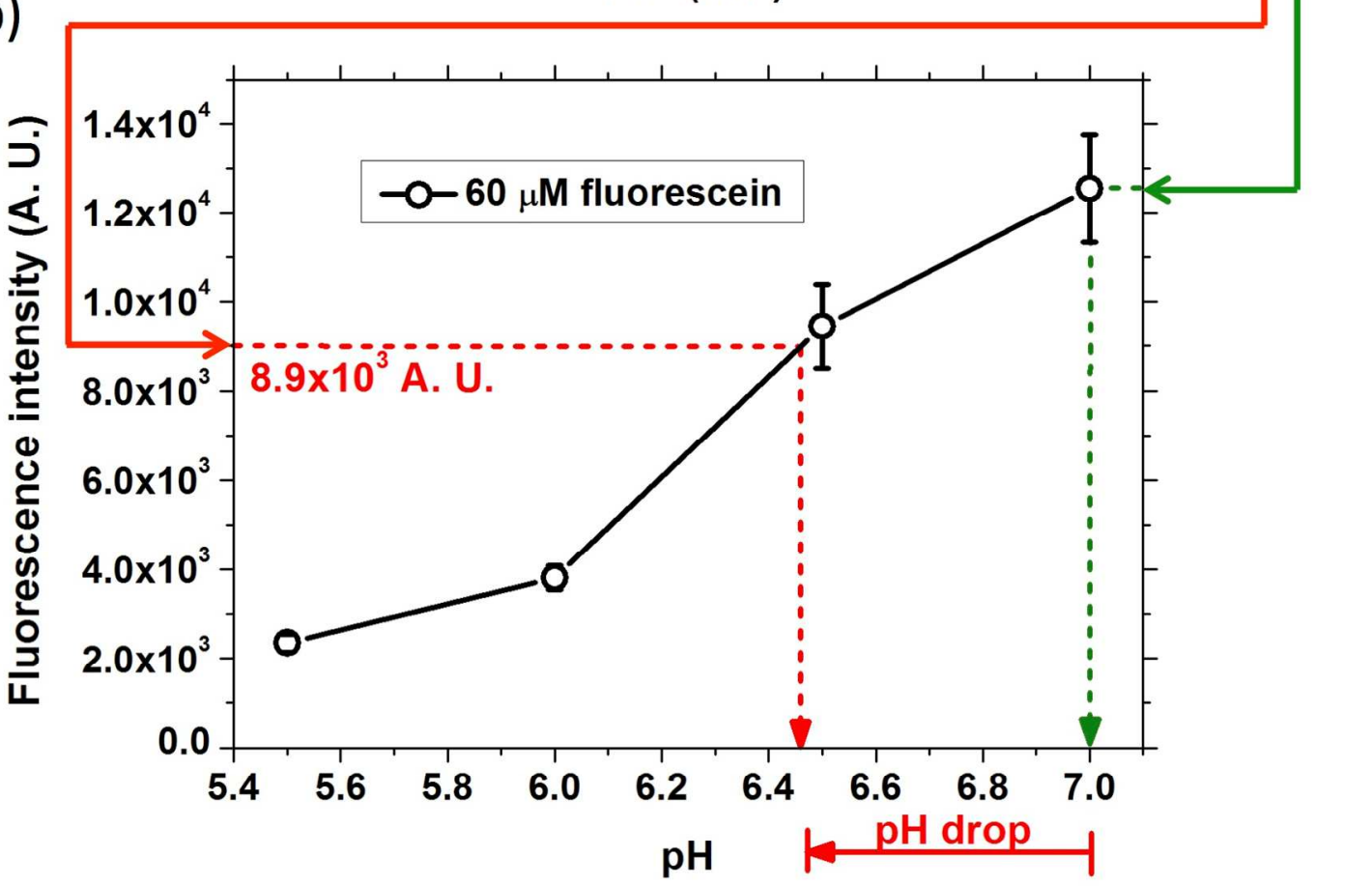

Figure S-5. Estimation of $\mathrm{pH}$ drop during electrokinetic concentration. (a) $50 \mathrm{~V}$ were applied to concentrate $0.1 \mu \mathrm{M}$ Alexa Fluor 488 and fluorescein (both fluorescent dyes were diluted in 0.1X PBS, pH 7.0) for $15 \mathrm{~min}$. in separate channels. The fluorescence signal intensity was acquired throughout the entire concentration period (exposure time was $100 \mathrm{~ms}$ and interval between each measurement was $30 \mathrm{~s}$ ). 
The linear approximations for the first $8 \mathrm{~min}$. of concentration are depicted as the dash-dot green lines. A concentration enrichment factor of $\sim 600$ times (concentration increased from $0.1 \mu \mathrm{M}$ to $60 \mu \mathrm{M}$ ) was measured for $\mathrm{pH}$-insensitive Alexa Fluor 488. The deviation from the linear regression line observed in the case of fluorescein results from its $\mathrm{pH}$ sensitivity. (b) To estimate the $\mathrm{pH}$ shift during electrokinetic concentration, $60 \mu \mathrm{M}$ fluorescein in $0.1 \times$ PBS was titrated from pH 7.0 to 5.5 in $0.5 \mathrm{pH}$ unit steps (fluorescence signal intensity between the experimental data points was linearly interpolated). From the comparison of the fluorescence signal intensity after $15 \mathrm{~min}$. of electrokinetic concentration [Fig. S-5 (a), red dot at 15 min.] with the tiitration curve suggests a $\sim 0.5 \mathrm{pH}$ drop from pH 7.0. 


\section{S7. Simultaneous DNA concentration in $\mathbf{5}$ channels}

Video S-5 shows a 15 min. simultaneous preconcentration of the DNA sample next to PEDOT:PSS membranes printed on top in five different microchannels. After dispensing $100 \mathrm{nM}$ DNA sample into the right reservoir, and the buffer solution into the left reservoir, the DNA sample was concentrated by applying 75 $V$ across the channels. As can be seen in the movie, the DNA plugs in different channels formed at the same rate and remained close to the membranes during the whole 15 min period. Once the voltage was switched off, all the DNA plugs moved away from the membranes. 


\section{S8. Channel-to-channel and device-to-device variation}

Channel-to-channel deviation is low because the average fluorescence intensity and standard deviation values of the DNA plugs and hybridization results are similar among the different channels. The chip-to-chip reproducibility (4 multichannel devices tested in total) is high since in general the error bars are small in comparison with the average fluorescence intensity values for both the DNA plugs and hybridization results. In fact, for $100 \mathrm{nM}$ : the average intensity concentration was 173040 and the standard variation was 41025 ; the average hybridization intensity was 4206 and standard variation was 819 . For $10 \mathrm{nM}$ : the average intensity concentration was 780 and the standard variation was 232; the average hybridization intensity was 97 and standard variation was 23 .

Table S-1 Measurement of the chip-to-chip reproducibility for PEDOT:PSSPDMS devices.

\begin{tabular}{|c|c|c|}
\hline $\begin{array}{c}\text { Concentration } \\
\text { (nM) }\end{array}$ & $\begin{array}{c}\text { Plug intensity } \\
\text { (A. U.) }\end{array}$ & $\begin{array}{c}\text { Hybridization signal } \\
\text { (A. U.) }\end{array}$ \\
\hline 100 & $173040 \pm 41025$ & $4206 \pm 819$ \\
\hline 10 & $780 \pm 232$ & $97 \pm 23$ \\
\hline
\end{tabular}




\section{References}

(1) Chen, A. K.; Cheng, Z.; Behlke, M. A.; Tsourkas, A. Anal. Chem. 2008, 80 (19), 7437-7444.

(2) Emmart, E. W. Arch. Biochem. Biophys. 1958, 73 (1), 1-8. 